\title{
On Novel Aspects of Hydrogen Effects on Applied Stress - Coupled Micromagnetic Activity in a Mild Steel After Exposure to $\mathrm{NaCl}$ - Water Solution: A Combined Approach
}

\author{
Alexandros Altzoumails ${ }^{1 *}$, Victor Kytopoulos ${ }^{2}$ \\ ${ }^{1}$ School of Chemical Engineering, National Technical University of Athens. Athens, Greece \\ ${ }^{2}$ School of Applied Mathematical and Physical Sciences, National Technical University of Athens. Athens, Greece
}

Received: June 17, 2021 Revised: July 30, 2021. Accepted: August 3, 2021. Published: August 11, 2021.

\begin{abstract}
Hydrogen produced after exposure of a low carbon steel to corrosive $\mathrm{NaCl}$ - Water solution may affect various its tensile mechanical and magnetic microstructural behaviour in a complex manner. This was investigated by introducing a relevant micromagnetic specific emission (ME) - response of this ferromagnetic material, where related processes and parameters of micromagnetic activity and mechanical response were implemented. In this manner, it was demonstrated that an increase in the hydrogen accumulation with corrosion time leads to an associated increase in the embrittling effect expressed by a substantial loss in the ductility of material. The competive and opposing effects of cumulative hydrogen, applied stress and plastic strain - induced microstructural damage were related to the specific MEresponse parameter by which an increased magnetic hardening tendency of material with corrosion time was possible to establish. In this fashion and by using a stress as well as strain mode of presentation- aided combined approach, the complex interplay between micromagnetic activity, hydrogen accumulation and applied stress-strain was better revieled and analysed. It was also shown that the embrittlement is a product of hydrogen accumulation introduced by two highly localized processes. As such, accumulation occurs in two characteristic parallel ways: one of a common lattice diffusion and one of hydrogen transport and redistribution by moving dislocation towards the affected sites. Concerning the highly localized effects the dominating role of hydrogen - induced damage in form void initiation and growth over the hydrogen assisted stress relief was reasonably demonstrated by using a simple modelling approach. Based on a mechanism of moving dislocation - assisted interaction between commulative hydrogen and magnetic domain walls, a Portervin - Le Chatelier - type micromagnetic process of a cooperative-corelated domain wall transport was proposed to explain certain subtle, quasiperiodic
\end{abstract}

behaviour of ME- response. In the frame of the above findings the superior sensivity of $\mathrm{ME}$ - response compared to the mechanical one in early detecting cumulative hydrogen - assisted microstructural damage changes can be d educed.

Keywords: J - Parameter, Hydrogen, Embrittlement, Void, Domain Wall, Pinning Strength, Dislocation, Stress- Strain.

\section{INTRODUCTION}

It is generally accepted that steel can absorb hydrogen from corrosive natural environment, production and processing and thus have its mechanical properties impaired, due to hydrogen - induced microcracking processes resulting in a more general phenomenon which is known as hydrogen embrittlement (HE). The problems of HE coupled with hydrogen - induced interfacial damage or microcracking has been of great importance of many loaded structural steel components of industries, such as power generating and marine constructions components. for example in situation where marine environment is encountered the possibility of HE arises because as it is known sea water - iron system forms an environment potential for atomic hydrogen production and diffusional transport into the material [1- 8]. It is also believed that molecular hydrogen is dissociated by chemisorption on iron surface allowing the liberated atomic hydrogen to diffuse internally and embrittle the metal [7]. Likewise, it has been shown that hydrogen is a product of corrosion reaction between iron and water [6]. This hydrogen then, follows the same path as chemisorbed hydrogen to the metal interior [5]. There is a considerable evidence in the literature that atomic hydrogen may segregate by rapid diffusion at internal particle - matrix interface, grain boundaries, microvoids and stress gradient sites by lowering the cohesive atomic bounding at these sites [5]. This in turn results in formation of extensive 
regions of voids and internal new inter faces. Furthermore, there is also evident that in addition to the interfacial site, hydrogen resides in traps such as dislocations and point defects, debris left behind the jogs of dislocation as they move through the metal during plastic deformation [4 - 6]. During plastic deformation, hydrogen appears to affect the mechanical properties of metal in a more specific way by controlling the ease by which dislocations nucleate and move through the crystal lattice. Many models suggested at this problem require the redistribution of hydrogen during the test. Especially, during slow - strain - rate tests at ambient temperatures hydrogen transported by moving dislocations, may essentially contribute to this redistribution $[8,9]$. Calculations in this direction have shown that hydrogen transport rates associated with dislocation motion can be several orders of magnitude greater than those associated with lattice diffusions [6]. In this aspect it has been shown that hydrogen affects the plastic flow by promoting the onset of localized plastic instability and so the premature fracture [6]. In general, the research on hydrogen - assisted cracking, in the last decades, has focused on two basic types of problem groups. One of them emphasizes the engineering - macroscopic aspects of evolution of strength parameters. The other group emphasized microscopic aspects where many of the above - mentioned problems are examined by obtaining certain correlations between microstructure and hydrogen - assisted microcracking mechanisms. This can be attained for example by means of combined microscopic experimental approaches of Scanning Electron Microscopy (SEM) - fractographic analysis techniques $[4,6,8-13]$. However, today, despite extensive research on these two problem groups a fully understanding the complexity of hydrogen - assisted microstructural damaging processes is not possible $[4,6,14$, 16]. Because of this, new variant aspects and approaches in the direction of mechanical and physical characterization of mechanically loaded steels after their exposure to corrosive hydrogen environment would be of significant importance for many structural steel components of various industries. Today, micromagnetic emission is a well-established, versatile and sensitive non - destructive testing method for a characterization of ferromagnetic steels at microscopic level where by using a well-known related micromagnetic emission phenomenon certain mechanical and microstructural changes in the material can be detected and analysed $[17,18,20]$. As such, this testing method may be used to give complementary and/or supplementary valuable information about the material. In the present complex study an effort is made to perform this task by employing the intrinsic micromagnetic emission phenomenon, known also as Barkhausen noise, of a tensile stressed low - carbon steel after its exposure to corrosive environment of $3.5 \% \mathrm{NaCl}$ - water solution. In this way, due to the microscopic character of the micromagnetic emission phenomenon, certain valuable macroscopic and microscopic combined aspects and approaches for some of the above mentioned problems are presented and analysed.

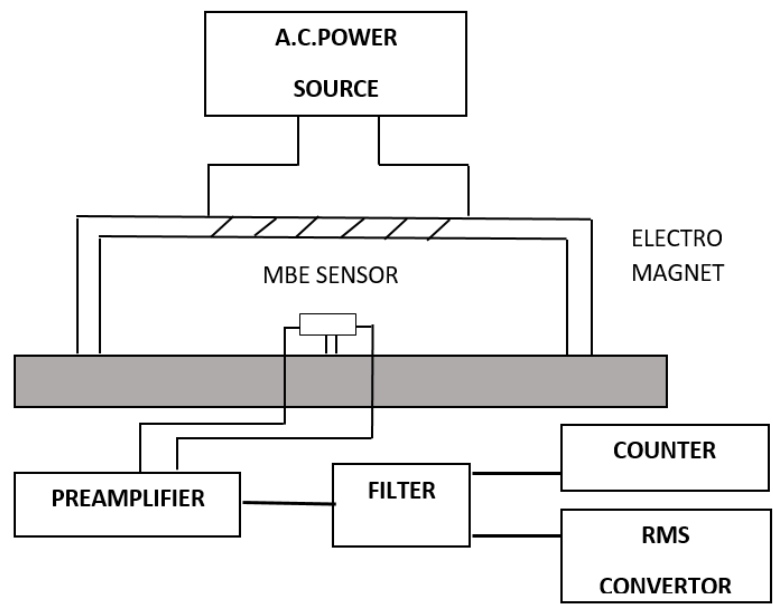

Figure 1. Block diagram of the experimental MBE - setup.

\section{THEORETICAL CONSIDERATION}

\section{A. General Assumptions}

It has been shown that atomic hydrogen and/or ion $\mathrm{H}^{+}$, may be a product of electrochemical reaction between iron and water and as such due to its very small size is capable to diffuse very rapidly through the crystal lattice into the bulk of the material $[1,2,4,5]$. Calculations of potentially greater significance have shown that hydrogen transport rates in association with dislocation motion may be several orders of magnitude greater than that associated with common lattice diffusion $[6,8]$. Thereafter, the hydrogen atoms (ions), due to their directional flow, tend to accumulate at various stress gradient sites such as grain boundaries, dislocation tangles or arrays, inclusions, voids, solute atoms and in general at internal interfaces. For example the directional flow controlled accumulation is caused by long - range elastic stress fields as well as electrostatic short range field interactions between hydrogen ions and dislocations. The electrostatic interactions take place along the dislocation core, where an excess of positive or negative pressure variation on edge dislocation leads to a rearrangement of the conduction electrons which will tend to move from compression to tension region. The electronic charge of hydrogen ion then interacts with the field of dipole, where the charge of the dipole is proportional to the elastic distortion of the lattice. It should be noted that the electrostatic interaction in b.b.c. metals is relatively high and roughly equals to the Cottrell atmosphere formation energy [19]. Hydrogen, accumulated in this way may induce several mutual, parallel effects which may have an influence on the micromagnetic activity response determined by changing the magnetoelastic and magnetostatic interactions of domain walls with various pinning (obstacle) sites such as voids, grain boundaries, dislocations tangles, inclusions, microcracks etc. Such an effect is the stress gradient relief due to mechanisms of hydrogen - assisted reduction in the interatomic cohesion force at electron level $[5,6]$. However, this mechanism is very complex and is not fully understood requiring special quantum mechanics calculations which can 
describe changes in chemical bounds caused by hydrogen [5]. A second and parallel effect is the hydrogen - assisted strain localization where nucleation and growth of voids at internal interfaces takes place $[8,13]$. Such interfaces are created for example around cementite $\mathrm{Fe}_{3} \mathrm{C}$, impurities or other inclusions as a result of decohesion of the inclusion - matrix interfaces. A third combined effect is related with micromagnetic activity mechanisms by which hydrogen-assisted growing voids with appropriate size may create new closure magnetic domain by which local domain refiment and reorganisation takes place [21-24]. Thereafter, on the basis of a detailed micromagnetic analysis concerning the pinning interaction field and forces (strength) between magnetic domain walls and inclusions or volume defects presented in $[21,22]$ it is possible to establish a reasonable operational approach as depicted in the working sketch of Figure (2), by the solid line curve [21,22]. This curve shows the behaviour of the void or inclusion - controlled pinning strength (force) degree $F_{p}$, with corrosion exposure time. As such, by this approach the behaviour of this strength may conveniently be adjusted and scaled to the changes of void size with corrosion time. To due this we should make some basic assumptions as given in the following:

$\bar{d}=\frac{d_{0}+\delta v}{\mathrm{dw}}$, where $\bar{d}$ is the reduced effective diameter of pinning obstacle and $d_{0}$ the effective diameter of the preexisting inclusions and precipitates and other microstructural inhomogeneities, or volume defects. It should in general be added that second phases, disperse precipitates, various inclusions, (say non-metallic), and non-uniformly distributed stresses and strains in microvolumes of a body are related to volume defects. In this sense the basic parameter, $d_{0}$, in the above formula may also represent several plastic strain - caused defects in form of tangled dislocations and microcracks around which hydrogen diffuses and is accumulated by creating interfacial defects in from of void clouds or atmospheres configurations. Further, $\delta v$ is the spacing created mainly between particle and crystal matrix due to the void - assisted interface decohesion, as shown in the sketch of Figure (3), and $d_{w}=65 n m$ is the theoretical thickness of the Bloch wall in iron. By means of SEM-aided metallographic measurements of the used steel an average value of about $\mathrm{d}_{0}=0.2 \mu \mathrm{m}$ for cementite and other inclusions and precipitates was abtained in this study.

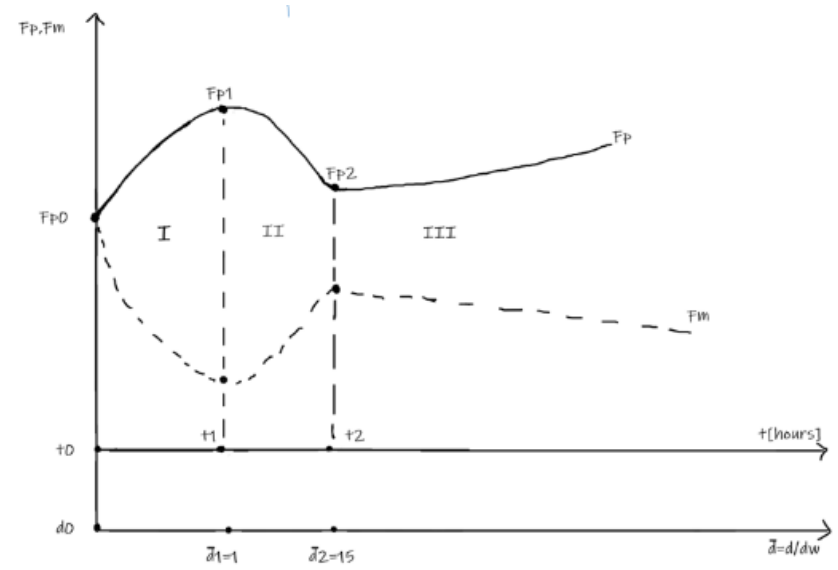

Figure 2. Sketch of pinning strength-affected regions by hydrogen accumulation

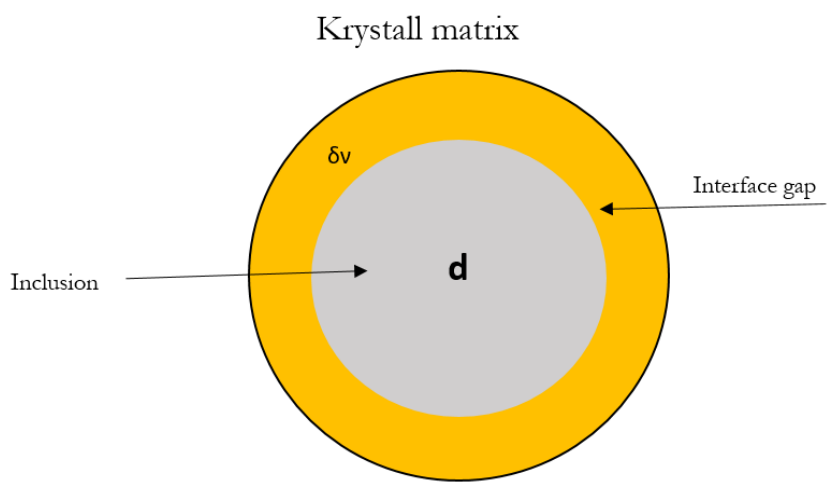

Figure 3. Sketch of matrix-inclusion interface created by void accumulation.

As shown in the working sketch of Figure (3), the pinning behaviour may roughly be characterized by three basic regions I, II, and III. Here, the double abscise expresses on the one hand the corrosion time in hours and on the other the reduced void (defect) diameter, created by hydrogen accumulation taking place during corrosion time. The increase in the pinning strength in the region I is due to the increase in the number of small-sized voids, around which magnetic free pole charges appear. This lead to the consponding increase in the magnetic interaction energy between domain walls and these microvoids. During the stage II, beyond the exposure time $t_{2}$, the number of earlier formed microvoids remain mostly constant but they increase in size. As such, a redistribution of the magnetic free poles occurs on wider surfaces thereby reducing the magnetostatic energy and hence the interaction between domainene walls and voids [22]. Consequent, a net reduction in the pinning strength or an increase in the mobility may result. The data given in Figure (2), are evaluated by $\bar{d}_{3}=$ $\bar{d}_{c r} / d_{w}=1 \mu \mathrm{m} / 65 \mathrm{~nm}=15 \quad$ by, $\bar{d} \mathrm{cr}=1 \mu \mathrm{m}=\mathrm{do}+\delta v=0.2 \mu \mathrm{m}+\delta v$ and hence $\delta v \cong 0,8 \mu \mathrm{m}$. The last value expresses an indicative size of the interface spacing (gap) between inclusions and matrix created by void growth. This size is comparable to the maximum average size of voids estimated by us however, such voids could not be observed by SEM in the present study. The formation of such large void structured interfaces under present mildly corrosion condition of $3.5 \% \mathrm{NaCl}$ - water solution and maximum corrosion time of $1000 \mathrm{hrs}$ might be admitted only with some reservation. This is because, such "large interfaces" would most probably be formed under applied stress and "hard" corrosive environment condition of high pressure gaseous hydrogen accumulation or cathodic hydrogen charging experiments [9, 10]. Furthermore, according to existing analysis approaches one can evaluate a critical obstacle (void) size $\bar{d}_{c r}=1 \mu \mathrm{m}$ above which extended transverse magnetic, spike-like, domains appear [21, 22]. Thus, the simultaneous increase in the void (accumulation) and transverse domain should favor an increase in the pinning strength. This is because the large voids, acting as obstacles, as well as the internal magnetic friction between basic domain and transverse ones would create a combined effect of pinning strength increase. Therefore, from the above mentioned one could expect that the initiation of region III could occur for times of corrosion of the order of maximum time given by experimental conditions of 
the present study. As such, the formation of the range III would show an increasing pinning strength (force) with time of corrosion. Thus, in a first approach, the first region could extend up to the reduced diameter $\bar{d}_{1} \simeq 1$ associated with time $t_{1}=200 \mathrm{hrs}$ at which, as it will be shown in the following discussion, the formation of a maximum $\mathrm{F}_{1}$ of pinning strength parameter $F_{P}$ can be estimated. Beyond this time, the experimental data allow to estimate that this parameter should fail up to a minimum value $\mathrm{F}_{2}$ for corrosion time $t_{2}=t_{\max }=$ 1000 hrs (region II). This decrease should be attributed at first to the formation around void- structured interfaces, microcracks and cavities of certain opposing stray fieds and auxiliary-closure magnetic domains in changing the pinning strength [24-28]. For times $t>t_{2}$, associated with the reduced void diameter $\bar{d}_{3}>15$ (region III), the pinning strength degree should again increase. This increase is expected, although with some reservation, to attain a saturation level for enough long exposure times. Nevertheless, it would be very interesting to investigate this behaviours for such long times and for this reason a related study is under preparation. Further, for sake of completeness it is assumed that $\mathrm{F}_{\mathrm{P} 1}>\mathrm{F}_{p 2}>F_{\mathrm{P} 0} \gg F_{p 1}-$ $F_{p 2}$, which means that for the present relative short experimental times the hydrogen - induced changes in the pinning strength are smaller compared to the initial, hydrogen - free pining state $F_{p o}$.

Furthermore, for the convenience of the following discussion and by means of the working sketch of Figure 2 one can construct the "inverse" of the pinning strength parameter denoted $\mathrm{Fm}=1 / \mathrm{F}_{\mathrm{P}}$ which now should express the degree of mobility of the magnetic walls. This is shown in Figure 2, by the interrupted curve which will be used as an additional operational parameter.

Consequently, the sketched regions in Figure 2 may generally be characterized as follows:

a) Region I, by increasing pinning strength or decreasing wall mobility, controlled by small - sized void systems formation

b) Region II, by decreasing pinning strength or increasing wall mobility controlled by medium - sized void systems formation.

c) Region III, by increasing pinning strength or decreasing wall mobility, controlled by large - sized void systems formation.

At this place it is suggested to explain again that an increase (decrease) in the wall mobility facilitates an associated increase (decrease) in the measured $\mathrm{V}_{\mathrm{rms}}$ - energy signal of ME - response [17, 18, 20]. This is because an increase (decrease) in the wall mobility is connected with the associated increase (decrease) in the effective wall velocity between pinning sites, fact that implies a corresponding increase (decrease) in the rate of change of magnetic domain volume [20, 27]. This rate of change in turn leads to a corresponding increase (decrease) in the measured signal $\mathrm{V}_{\text {rms. }}$.

\section{B. Specific Assumptions}

A more specific and subtle hydrogen effect is the change in the lattice spacing caused by occupying an interstitial hydrogen atom of a tetrahedral interstice resulting in a change in the crystal lattice spacing. This spacing is one of the most important crystallographic parameter and as such is coupled with, the mostly basic magnetic parameters as follows [21-22]

$$
A=\frac{3 \mathrm{KTcSc}}{2 a_{0}(s+1)}
$$

where A is the Heisenberg exchange energy, Tc is the Currie temperature, $K$ is the Boltzmann constant, $S$ the spin quantum number of ferromagnetic electrons per atom, $a_{0}$ the crystal lattice spacing and $c=1 / 4$ for b.c.c metal lattice. Furthermore, concerning the micromagnetic structure the following relationships hold [21-23]

$$
\begin{gathered}
d_{w}=3 \times 10 \times\left(\frac{\gamma_{w} \cdot l}{I_{s}}\right)^{1 / 2} \\
\gamma_{w}=4\left(\mathrm{AK}_{1}\right)^{1 / 2} \\
\delta w \simeq \sqrt{A / K_{1}}
\end{gathered}
$$

Where $d_{w}$ is the domain width

$\gamma_{w}$ the specific wall energy

$I_{s}$ the saturation magnetization, $\delta \mathrm{w}$ Bloch wall thickness,

$l$ average crystal grain length, and

$K_{1}$ magnetocrystalline anisotropy energy constant

Now, a combination of the above formulae (1), (2), (3) yields

$$
d_{w} \sim \gamma_{w^{1 / 2}} \sim \frac{K_{1}{ }^{1 / 4}}{a_{0^{1 / 4}}}
$$

In presence of initial (internal) distortional stress and strain fields of the crystal lattice the last formula becomes an important one as given by

$$
d_{w} \sim \gamma_{w^{1 / 2}} \sim \frac{K_{\mathrm{eff}}{ }^{1 / 4}}{a_{\mathrm{eff}^{1 / 4}}}
$$

Where

$$
K_{\mathrm{eff}}=K_{1} \pm \frac{3}{2} \lambda_{s} \sigma_{0}
$$

is the effective or equivalent magnetocrystalline anisotropy, $\lambda s$ the saturation magnetostriction and $\sigma_{0}$ the internal- effective distortional stress.

Yet:

$$
\bar{a}_{\mathrm{eff}}=a_{0} \pm \Delta a
$$

is the effective lattice spacing constant and $\Delta \mathrm{a}$ the lattice distortion.

Furthermore, by combing of eqns. (2), (3) an (3a) one obtains:

$$
\frac{1}{d_{w}} \sim\left(a_{0} \cdot \delta_{w}\right)^{1 / 2}
$$

where $\delta w$ is the Bloch wall thickness of magnetic domain.

Now, in presence of internal long - range stress, as in case of dislocations tangles or cells the micromagnetic theory of permeability allows to introduce an operational interaction 
pinning force (strength) parameter [21-22], as given by:

$$
R \sim\left(\mathrm{N} / \delta_{w}\right)^{1 / 2}
$$

where $\mathrm{N}$ is the current dislocation density which remains constant during hydrogen accumulation process or corrosion time.

By combining of both of the above formula 9 and 10 one obtains:

$$
R / d_{w} \sim \sqrt{a_{0}}=\text { Constant }
$$

This seems plausible since the lattice spacing under the influence of hydrogen accumulation on interstitials may change very little [13]. Because of this and bearing in mind equation 10 and the low power exponent of eqn. (6), only a very limited change in the domain width $d_{w}$ can be expected. This in turn, due to the last equation (11) implies a corresponding limited change in the operational pinning force (strength) parameter R of eq. (10). Consequently, a stress relief effect caused by hydrogen accumulation would have a secondary influence on the strength of pinning sites. This means that any contribution to considerable changes in pinning strength should come at first from hydrogen assisted void growth process, fact consistent with earlier explanations regarding the working sketch of Figure (2).

\section{EXPERIMENTAL PROCEDURES}

Magnetization in ferromagnetic materials is due to the nucleation and growth of domains which result from creation and motion of domain walls with increasing external exitation magnetic field. The motion of domain walls can be often pinned by microstructural inhomogeneities such as precipitates, dislocation tangles and several lattice defects existing in the material and results in their discontinuous motion. As a result of this kind of abrupt domain motion, a pulsating induced voltage can be measured associated with the rapid change of magnetic volume due to interrupted jumping displacement of domain walls. This phenomenon is called the micromagnetic emission effect known in the literature also as Barkhausen noise. Further details concerning the used instrumental Barkhausen set-up are given in [25]. A block diagram of the experimental set up used for the measurement of micromagnetic Barkhausen emission (MBE) is shown in Figure 1 . The specimen had dogbone - type geometry where thickness was $2 \mathrm{~mm}$, width $10 \mathrm{~mm}$ and the effective gauge length $100 \mathrm{~mm}$. The samples were subjected to uniaxial tensile test at room - temperature and nominal low strain rate $10^{-4} / \mathrm{s}$, using a universal testing machine of Intsron - type. The ultimate stress was $380 \mathrm{MPa}$ and the yield stress ( $0.2 \%$ offset) was $190 \mathrm{MPa}$. Before testing, the samples were exposed to a corrosive environment produced by a continuously sprayed $3.5 \% \mathrm{NaCl}$ aqueous solution in a Salt Spray Fog (SSF) apparatus. All corrosion experiments were performed at room temperature $\left(25 \mathrm{c}^{0}\right)$. The exposure time in the SSF- apparatus were $200,400,600,800,1000$ hours. The final corrosion product formed on the specimen surface was in a greatest part, as obtained by $\mathrm{X}$ - ray diffraction analysis, a ferric oxyhydroxide (FeO-OH) component. The produced corrosion layer was removed by dry air blast and soft natural bristle brush revealing underneath a black and strong adhering. It is pointed out that during the corrosion production of atomic hydrogen takes place by the following basic electrochemical reactions [1]:

$$
\begin{gathered}
\mathrm{Fe}=\mathrm{Fe}^{+2}+2 \mathrm{e}^{-}, \\
\mathrm{Fe}^{+2}+2 \mathrm{FeO}(\mathrm{OH})=\mathrm{Fe}_{3} \mathrm{O}_{4}+2 \mathrm{H}^{+}, \\
\mathrm{H}^{+}+\mathrm{e}^{-}=\mathrm{H}
\end{gathered}
$$

The chemical composition of the low-carbon steel used in this study is given in Table 1

Table 1. The chemical composition of used low-carbon steel.

\begin{tabular}{lllllllll}
\hline $\mathbf{C}$ & $\mathbf{M n}$ & $\mathbf{S i}$ & $\mathbf{N i}$ & $\mathbf{C r}$ & $\mathbf{M o}$ & $\mathbf{S}$ & $\mathbf{P}$ & $\mathbf{N}$ \\
\hline 0.04 & 0.44 & 0.015 & 0.016 & 0.015 & 0.002 & 0.016 & 0.02 & 0.004 \\
\hline
\end{tabular}

\section{RESULTS AND DISCUSSION}

\section{A. Strain-Mode of Presentation}

It is known that for several investigations using the ME response technique among various measuring parameters the common count rate and corresponding root-mean-square voltage $V_{r m s}$ signal are preferred $[17,18,20]$. Nevertheless, some apparent "problems" may arise by using these parameters separatelly. This is because the $V_{r m s}$ reflects the qualitative behaviour where the number of counts $\mathrm{N}$ the quantitative one of micromagnetic activity. For example, by comparison of the obtained count (pulse) rates and the corresponding $V_{r m s}$ signal curves shown in Figure (4), certain "inconsistencies" may arise in the sense that for certain strain point or regions the two signal curves show different trend of behaviour. For instance one can observe strain regions where a decrease in the count signal is associated with an increase in the $V_{r m s}$ - signal. As such, this would introduce certain ambiguities in the interpretation of the obtained data. To overcome, at least in part, this "inherent weakness" we propose to use an operational variable parameter $\mathrm{J}=V_{r m s} / \mathrm{N}$, where $V_{r m s}$ is the measured root- mean- square voltage and $\mathrm{N}$ the corresponding detected total number of counts (pulses). In this aspect the following relationships may be assumed: $\frac{V_{i n_{i}}}{V_{r m s}}=J_{i}, \sum_{i} J_{i}=J, \quad \sum_{i} V_{i} \cdot n_{i}=V_{r m s}$ and $\Sigma_{i} n_{i}=$ $N$. This results from Pulse Height Distribution measurements given elsewhere, according to which, a given group of $n_{i}-$ domain walls may induce, at a given instant time, the associated group voltage $V_{i}[18,20]$. Thus the $J_{i}$ parameter can be seen as a measure of the strength of group and as such $\mathrm{J}$ can reflect an overall specific energetic ME - response. It should be added that the mentioned specific energetic character of the proposed $\mathbf{J}$ - parameter includes the response of nucleation and growth of reverse magnetic domains during a magnetization cycle. This is because any local modulation of magnetic anisotropy strength or directions around inhomogeneities can lead to premature domain nucleation and annihilations or switching events. If such events early happen a low energy domain state is occupied and magnetization 
process continues by discrete jumping displacement processes [23].

It has been observed that an increase in the applied magnetizing field would lead to an effective domain wall multiplication, associated with a decrease in the effective wall velocity and hence in the induced signal energy [27]. From these factors one can reasonably deduce that an increase in the number, $\mathrm{N}$, of pining sites (density), would result in an decrease in the ratio of J-parameter. However, this happens only for an applied stress-free state of the material. As it was shown in a recent study this behaviour of the $\mathrm{J}$-parameter may change substantially for a stressed material [25]. Further, we have was observed that the $\mathrm{J}$ - parameter may be a robust and characteristic experimental variable for the material in the sense that for the same stress conditions it may remain almost constant by changing the measurement locations on the specimen surface and especially by including the mostly inevitable lift - off effects simulated by varying the inclination angle between the pickup probe and specimen surface. This was concluded from statistical estimation of data where the standard deviation was taken as the uncertainty criterium [18]. As such, it was observed that for a set of given number of measurent surface points and a set of repeat measurements independent on electronic settings on the apparatus such as signal gain and/or threshold signal levels. Since of changing by $\pm 15^{\circ}$ the inclination angle from the normal position of probe to specimen surface, the obtained uncertainty was about five times lower than those obtained by taking Vrms and the number of magnetic counts $\mathrm{N}$ separately. Further, it was also observed that the specific parameter $\mathrm{J}$ is also almost this parameter seems to have a certain specific physical meaning and may be influenced by the investigated mechanical and physical factors such as micromagnetic changes, cumulative hydrogen as well as stress-strain-induced microstructural changes, it will be assumed in the following to describe the general physicomechanical behaviour of steel under these conditions. Moreover, in a more specific magnetic aspect the proposed $\mathbf{J}$ - parameter could also be an indicator of the current states of the ME - "spectrum" changing from numerous, low energy wall jumps (small pulses) described by a decreasing $\mathrm{J}$ - parameter, to view, high energy wall jumps (large pulses), described by an increasing $\mathrm{J}$ - parameter [30]. In this context, it is suggested to presume that an increasing (decreasing) $\mathrm{J}$ - parameter may indicate an associated decreasing (increasing) of magnetic hardening tendency of the steel. As such, the magnetic hardening of steel seems to be an important micromagnetic property which, as it will be shown latter, can be related with its hydrogen - assisted embrittlement process.

The representative series of Figs. $5-9$ were obtained by a recent study [25]. The change of $\mathbf{J}$ - parameter with strain of the corrosion - free and corrosion - affected material is presented. For convenience of the following discussion in each figure the corresponding engineering stress - strain curve of the material was inserted. At first, it is interesting to observe the formation of a maximum in the $\mathrm{J}$ - parameter curve, similar to that in the stress - strain curve. This fact implies to assume that certain related microstructural changes should occur for both curves at this maximum point. Thus for the stress - strain curve, at this point, the well - known phenomenon of plastic instability in form of a localized necking sets on, which is a precursor of ductile fracture. At the same time, at this point, a state of high triaxle stresses develops by producing various internal microstructural damaging changes in form of voids, cavities, and microscracks which by coalescence lead to the final - fracture of the material [6]. Because all of these, it seems logical to tray to analyse, for example as shown in Fig 5 , the response behaviour on the basis of two major characteristic stages; a prenecking stage I, and a postnecking stage II, separated by the critical strain point $\mathrm{e}_{\mathrm{c}}$ corresponding to the ultimate tensile strength value. At this place it is pointed out that all magnetic measurements were taken within the plastic necking region of the tensile stressed specimen. This was so, because in this manner elastic unloading effects occurring in the auteur regions of necking, can greatly be minimized. Otherwise, such effects would considerably affect the measured magnetic signal.

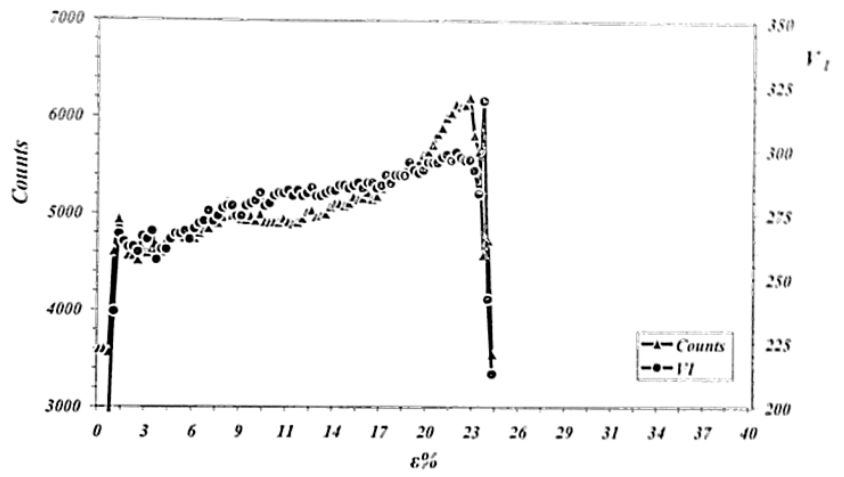

Figure 4. Plot of the Barkhausen emission signal of counts and volts respectively.

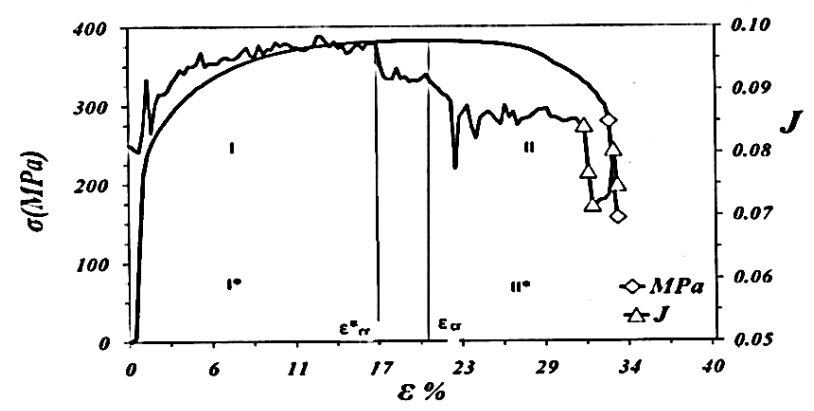

Figure 5. Mechanical stress-strain and magnetic $J$ - response curves for virgin (as received). 


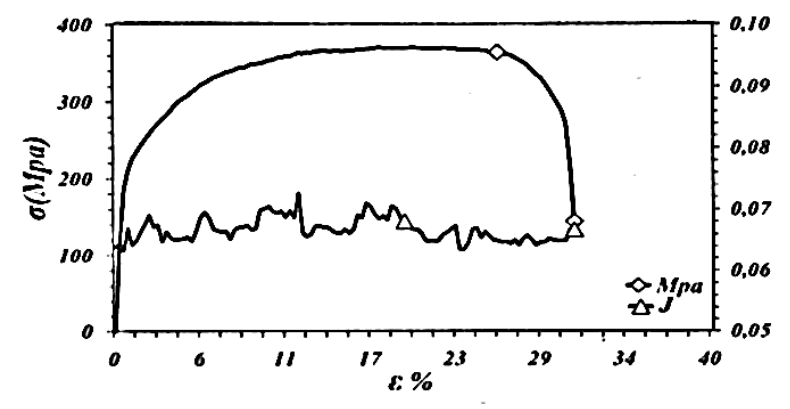

Figure 6. Mechanical stress-strain and magnetic $J$ - response curves for 200 hours of corrosion time.

Thus, it would be helpful and of valuable practice to tray to analyze, as shown in the same figure, the stress-induced micromagnetic response behavior on a basis similar to the above - introduced two characteristic mechanical response stages: prior magnetic stage $\mathrm{I}^{*}$ and posterior magnetic one $\mathrm{II}^{*}$, separated by the corresponding critical magnetic point $\mathrm{e}_{\mathrm{c}}{ }^{*}$. Thereafter, in Fig (5) an evident overall increase in the $\mathrm{J}-$ parameter, within the stage I* is observed, followed by a "drop" at $e_{c}{ }^{*}$ and close to $e_{c}$. This behavior can be attributed to a compending influence of applied stress and plastic strain on the magnetic response. Primarly, tensile stresses favor an increase in the magnetic energy signal $[17,18,20]$. At the same time, however, plastic strain - induced pinning sites in from of dislocation pile - ups and tangles, exhibit a pronounced suppressive effect on the magnetic energy signal [26]. From the above, the dominating influence of the applied elastic and flow stress over the plastic strain in increasing the $\mathrm{J}$ - parameter within the precursor stage $\mathrm{I}^{*}$, results. Beyond the characteristic magnetic point $\mathrm{e}_{\mathrm{c}}{ }^{*}$, and within the posterior magnetic stage II* the physicomechanical behavior is characterized by an evident overall decrease in the $\mathrm{J}-$ parameter of specific ME - response. It seems that, this time, within this stage, plastic strain - induced pinning effects dominate over the applied stress effects in decreasing the specific ME - response. Consequently, as earlier mentioned, a decreasing $\mathrm{J}$ - parameter is indicative of an increasing tendency of magnetic hardening. This is because, within the posterior magnetic stage, the behavior is controlled by the plastic strain - caused microstructural volume damage in form of microcracks, voids and other cavities which acting as additional pinning sites, can increase the number of measured counts $\mathrm{N}$. In this sense, the volume damage under applied stress conditions may act as a dense - spaced local triaxial stress raisers which lead to an increase in the magnetoelastic interaction energy with domain walls and hence the pinning strength. This afterwards, as earlier explained, results in a decrease in the effective wall velocity and consequently in the rate of magnetic volume change and hence in the induced $\mathrm{V}_{\text {rms }}$ or energy signal. As such the ration of the y-parameter subjected to a net reduction. Furthermore, for the sake of completeness of the previous discussion one should make some valuable comments on the "noise" inform of a more or less stochastic ripple observed in the J-curve of Fig 5. Thus, this "noise" formation in the J-curve should not be attributed to the common electronic noise of Barkhausen apparatus, but rather to the consecutive occurrence of opposing applied stress and strain effects, where as earlier mentioned, stress stimulates an increase whereas plastic strain a decrease in the specific ME response. In more details, the minute increase and decrease in $\mathrm{J}$ - parameter curve in form of "noise", reflex the associated process of consecutive pinning and unpinning- breakaway steps of a certain low portion of domain walls occurring around forming dislocation tangles and volume defects. This process becomes more intensive within posterior range II*, where "noise" or ripple amplitude is increased. This intensification could tentatively be explained, by the way where, due to the increased pinning strength (energy), the unpinning (detachment) of domain walls occurs at a higher start acceleration causing a corresponding higher average wall velocity and hence a net increase in the induced voltage [26].

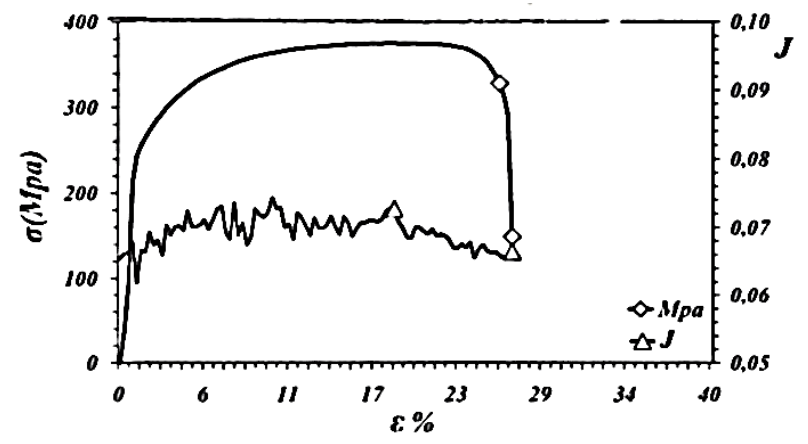

Figure 7. Mechanical stress-strain and magnetic $J$ - response curves for 400 hours of corrosion time.

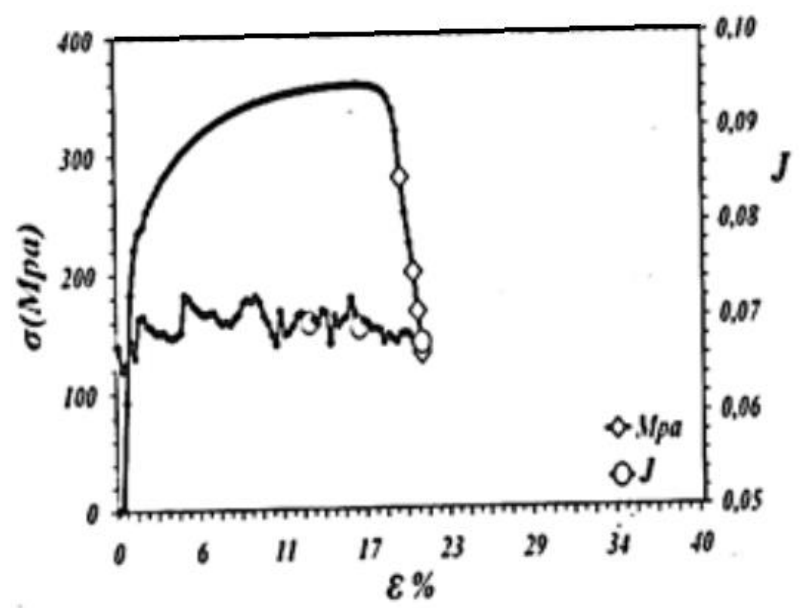

Figure 8. Mechanical stress-strain and magnetic J-response curves for 800 hours of corrosion time.

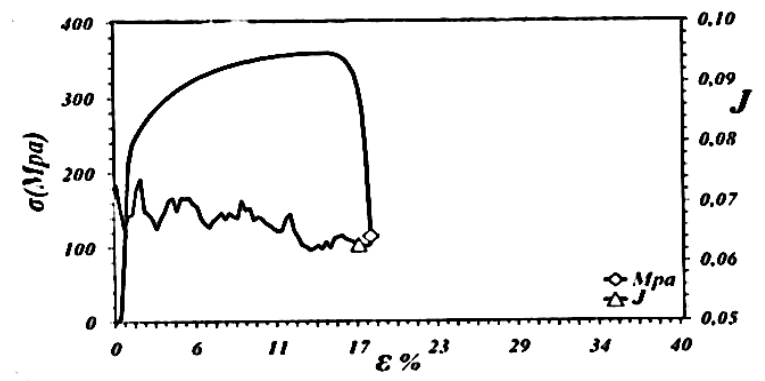

Figure 9. Mechanical stress-strain and magnetic J-response curves for 1000 hours of corrosion time. 
Based on the above constructive aspects and approaches concerning the specific $\mathrm{J}$ - parameter of the corrosion (hydrogen) - free material one can proceed in the following to present and discuss the behavior of cumulative hydrogenaffected material with corrosion time. Thus, by comparison of representative series of Figures. $5-8$ one can observe some distinctive differences which can be established by the following major factors:

i) Overall, vertical shift of all corrosion - related J- curves to lower magnetic values with increasing corrosion time, compared to corrosion (hydrogen) - free material.

ii) The maximalization of this shift is obtained for about $200 \mathrm{hrs}$ of corrosion time.

iii) Quasiperiodic small amplitude changes expressed by formation of more or less systematic ripple - type in Jparameter curves.

iv) Overall horizontal shift of $\mathrm{J}$ - parameter curves towards lower strain with corrosion time.

v) The position discrepancy between the critical strain points $e_{c}$ and the magnetic one $e_{c}{ }^{*}$ increases with corrosion time.

In the next, we try to discuss these factors one by one. The first mentioned factor of overall shift towards lower $\mathbf{J}$ - value with corrosion time was estimated by tacking as reference point the maximum of $\mathrm{J}$ - parameter curves.

This shift is indicative of the strong influence of the hydrogen accumulated during corrosion time on the physicomechanical behavior. As such, the dominating influence of hydrogen accumulation over the stress influence in lowering the specific micromagnetic response arises. At the same time, as earlier mentioned, a reduction in the $\mathrm{J}$ parameter indicates an increasing magnetic hardening tendency of the material. In general, if one try to correlate this tendency, denoted by $\mathrm{T}(\mathrm{J})$, with common permanent, hard magnetics, especially of high pinning - type [17, 24], than in a first approximation, one may reasonably assume $T(J) \frac{1}{F_{P}^{n}} F_{m}^{K}$, where $F_{P}$ and $\mathrm{F}_{\mathrm{m}}$ are the pinning force and wall mobility degree respectively and $\mathrm{n}, \mathrm{k}>0$. As such, hardening (softening) trend of the steel may roughly be described by this functional relationship.

Concerning the mentioned second factor, we use the measurements of the zero -strain and maximum lode of $\mathrm{J}$ parameter with corrosion time. The obtained data are presented in Figure 10, where the amazing qualitative similarity with the sketch of Figure 2 can be seen. As such one can observe that up to time $t_{1}=200 \mathrm{hrs}$ of corrosion a relative decrease whereas beyond this time a distinctive slow increase in this parameter occur. This behavior can reasonable be explained by the following arguments: During the corrosion period up to $200 \mathrm{hrs}$ hydrogen accumulation assists in formation of predominantly small - sized void structures around inhomogeneities, which according to region I of Figure (2) act as obstacles of increasing pinning strength or decreasing wall mobility.

For corrosion time larger than $200 \mathrm{hrs}$ the increasing hydrogen accumulation favorises the formation of predominantly "medium" - sized void structures around inhomogeneities, which according to region II of Figure (2) Act as pinning sites with decreasing pinning strength or increasing wall mobility.

By inspection the series of Figures (6-9) one may deduce that the formed ripple "modulations" in the J-curves show a more or less systematic evolution compared to the more or less "stochastic" one in the J-curve of Figure 4 concerning the corrosion-free material. The formation of ripple - type "modulations" of the corrosion $\mathrm{J}$ - curves is an interesting fact which can tentatively be explained by adopting a Portervin Le Chatelier - type micromagnetic Barkhausen activity of cooperative domain wall transport as follows: During plastic deformation a relative small portion of the total moving domain walls may become strong pinned i.e. locked in position for indefinite numbers of magnetization cycles at defect sites such as moving dislocations. The strong pinning arises because during slow plastic straining, atomic hydrogen has enough time to continue to be accumulated and distributed rapidly around these sites by means of its transport through moving dislocations resulting in formation of an excess in small - size void structures which, as mentioned earlier by means of region I of Figure (2), act as sites of increased pinning strength. In this instance the wall locking or immobilization time is long enough to allow further rapid inflow of atomic hydrogen into these sites by formation, this time, of Cottrell - Snoek - type atmospheres (clounds) structured by medium - size voids. As such, according now to region II of Figure (2), these sites in combination with dominating stress relief effects exhibit a decrease in pinning strength. This results in a net-minute, but critical reduction of the effective pinning strength thus allowing the domain walls, by unlocking (unpinning) or breakaway from their associated dislocation - atmosphere systems, to jump to the next moving pinning site. In other words one can argue that the above described process reflex a cooperative domain wall transport, promoted by combined moving dislocation and hydrogenassisted wall locking /unlocking steps. This process may be repeated (quasi - periodically) again and again resulting in a respective alternating increase and decrease in the related number of moving walls, macroscopically observed in form of ripple-type "modulation" of J - parameter curves with applied flow stress. As it was shown in a recent work.

Furthermore, the another interesting fact concerning the total shift of the $\mathrm{J}$ - curves with corrosion time towards lower strains is indicative of occurrence of hydrogen - assisted environmental embrittlement processes [25]. This shift is consistent with the total shift of the related (mechanical) stress - strain curves towards lower strains with time of corrosion as shown in Figures. (6-9) where an evident reduction in the ductility of the material can be deduced. It is mentioned that similar shifts due to hydrogen embrittlement have been obtained elsewhere $[3,28]$. With respect to this it is pointed out that a convincing evidence of occurrence of hydrogen embrittlement was gained by microscopic indicators of a detailed SEM - microfractographic analysis, while the phenomena of embrittlement is now to involve the interaction of hydrogen and defects, issues around prissily locating 
hydrogen atoms throughout the steel microstructure have hindered deeper investigations [28] . Thus, the combination of low level of interaction between hydrogen and electron, and the very high diffusional fugacity of hydrogen renders it externally difficult to experimentally determine its location by electron microscopy. Nevertheless, at this place it should be noted that there still exist problems with the full acceptance of hydrogen as universal embrittling agent of metals in corrosive salt - water environments, in the sense that the chloride agents - induced aggressiveness in this corrosive environment may play a certain important role [13]. Because of this, we have used the SEM - aided Electron Probe - X-ray Microanalysis of wavelength disperstion type technique for the fractured surfaces to detect chloride - type agents. Within the sensitivity limits of this technique, 100ppm, such agents could not be detected. Furthermore, it is known that chloride agents promoted electrochemical corrosion phenomena are related to long - time (months or years) - needed pitting formations on the materials surface. In our case, perhaps due to short corrosion times (maximum $1000 \mathrm{hrs}$ ), pitting features could not be observed. By virtue of these findings one can exclude any important role of such agents for the present investigated embrittlement processes. Nevertheless, the acceptance of hydrogen as the main embrittling agent impose to give an explanation of the phenomenon as follows: embrittlement, in general, denotes a tendency of premature fracture of a material by reduction its ductility. Thus, in our case, hydrogen flowing into dislocation core region leads to a weakening of crystalline interatomic or chemical bonds in this region, making so the dislocation more mobile by easy sliding along crystal planes at low stress levels. Consequently, at these stress levels high concentration of dislocation may early occur associated with highly localized plastic flow and high stress concentrations facilitating a premature fracture.

Finally, the mentioned last factor concerning the increasing discrepancy between the critical points $e_{c}$ and $e_{c}{ }^{*}$ with corrosion time may be interpreted as an advancing response of the second point for an early indication of critical microstructural changes. Thereafter, by taking these points as reference for the maximum of mechanical and $\mathrm{J}$ magnetic curves, one can observe the absolute shift of these points as well as the higher shift rate of the magnetic point towards lower strains with increasing corrosion time.

For instance, at $1000 \mathrm{hrs}$ of exposure to corrosion, as it is shown in Figure (9), the magnetic reference point of the Jcurve is shifted close to zero strain point, whereas the corresponding ultimate strength point has still an appreciable distance from this point. It can therefore be argued that the magnetic response may be in advance on mechanical one of the material. This in turn, suggests to accept that the magnetic testing, compared to the mechanical one, should have an increased sensitivity in early detecting hydrogen - assisted microstructural damages indicative of embrittlement occurrence in stressed steels.

\section{B. Stress - Mode of Presentation}

Now, further complementary and / or supplementary detailed and valuable information about the hydrogen influence on the investigated physicomechanical behaviour can be gained by the "stress - mode" of presentation as shown in representative series of Figures. (11-15). Compared to the previously discussed "strain - mode" of presentations, in this mode, the evolution scale of the prenecking stage of deformation is expanded at the expense of the postnecking stage. In this manner, the "stress - mode", as shown in the mentioned Figures, offers the opportunity to investigate in more details the physicomechanical behaviour during the prenecking stage, including elastic, microyield and early flow stress periods of loading. Thereafter, to give a more clear and concise explanations of the related phenomena, some basic reference points on the stress axis were selected. First, a "physical" yield point is introduced as follows: the Hall Petch relation, i.e. $\sigma *_{y} \sigma_{0}+k_{y} d^{-n}$, is used where $\sigma *_{y}$ is the stress to initiate plastic flow and $\sigma_{0}$ is the Pierls - Nabarro friction stress needed to move the initially immobile dislocations in slip planes, through the crystal [19]. The term $k_{y}$ is a dislocation locking constant associated with propagation of deformation across grain boundaries according to Cottrell's model [19]. Further, for low carbon steel it is valid that $k_{y}=24 \mathrm{~N} / \mathrm{mm}^{3 / 2}$ and $\sigma_{0}=72 \mathrm{MPa}$ [19]. In addition, the average grain size of the examinated steel was found to bed $\simeq 25 \mu \mathrm{m}$. Putting these data into the above Hall - Petch formula, one obtains roughly that $\sigma_{y} * 80 \mathrm{MPa}$ as the "physical" yield point of the material. Next, a flow stress point, $\sigma_{f} *$ corresponding to a strain above about $2 \%$ is introduced because at this strain, cell - structured dislocation network begins to develop [19, 29]. As a third reference point. $\sigma_{n} *$, is taken the necking stress corresponding to the ultimate strength (peak point) in the stress - strain curve. Thereafter, one can proceed in examination of the obtained data in the representative series of Figures. $(11-15)$. At first, in Figure (11), one can observe, for corrosion - free material, a quasiequilibrated behavior of the $\mathrm{J}$ - parameter, within the elastic stressing ie., below yield point $\sigma \mathrm{y}^{*}$. This is followed by an evident increase within the range of yielding $\left(\sigma_{\mathrm{y}}{ }^{*}\right)$ and cell flow stress point $\left(\sigma_{\mathrm{f}}^{*}\right)$. In the elastic range the applied tensile stress may influence the J-parameter of specific ME-response by several competing and opposite stress effects. First, the applied stress-induced magnetoelastic energy can be minimalized by domain wall multiplication or refiment in reducing the basic domain width $[18,20]$. This would lead to an increase the number of pinning/ unpinning events per time unit and hence to an increase in the rate of measured pulses (counts) N. At the same time, applied tensile stress may reduce the pinning energy height level by certain mechanisms where among others tensile stresses by disfavourising the transverse domains attached to supplementary closure domain, reduce the internal magnetostrictive friction and hence the effective pinning interaction between surface closers and basic domains $[20,23,24]$. In this sense, by this effect, a net reduction or relaxation of the effective pinning strength is caused. At the same time, tension tend to orient the domains close to the direction of applied stress thereby stimulating an increase in the Barkhausen emission energy. As such, by simultaneous increase in the measured voltage, $\mathrm{V}_{\mathrm{rms}}$, and the number of counts (pulses), $\mathrm{N}$, the ratio of the $\mathrm{J}$ - parameter may remain almost constant.

Furthermore, in the plastic range of loading beyond the 
yield point $\sigma y^{*}$ the dominance of combined effect of applied elastic and flow stress over the hardening plastic strain in increasing the $\mathrm{J}$ - parameter can be stated. This may reasonable be explained as follows: at the physical yield point $\sigma_{\mathrm{y}}{ }^{*}$, a process of dislocation motion and multiplication, localized within a grain, sets on. With further increase in the stress, dislocation motion and multiplication process is intensificated causing a process of an overall relay-race intergrain dislocation multiplication activity followed afterwards by a process of initiation of cell - structured dislocation network at cell flow stress point $\sigma_{\mathrm{f}}{ }^{*}$. Between these two processes a transitional stress state should exist, initiated by physical yielding and terminated by cell flow stress. Due to the strong magnetoelastic interaction between domain walls and this transitional state may trigger an appropriate correlated transport of domain walls by moving dislocations, macroscopically observed in Figure (11), in form of a transitional range of increase in the J-parameter $[29,30]$.

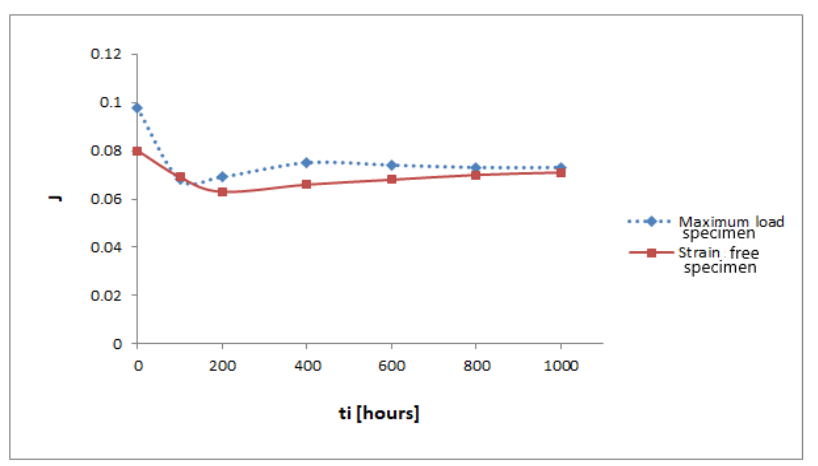

Figure 10. Magnetic J-parameter evaluation with time of corrosion.

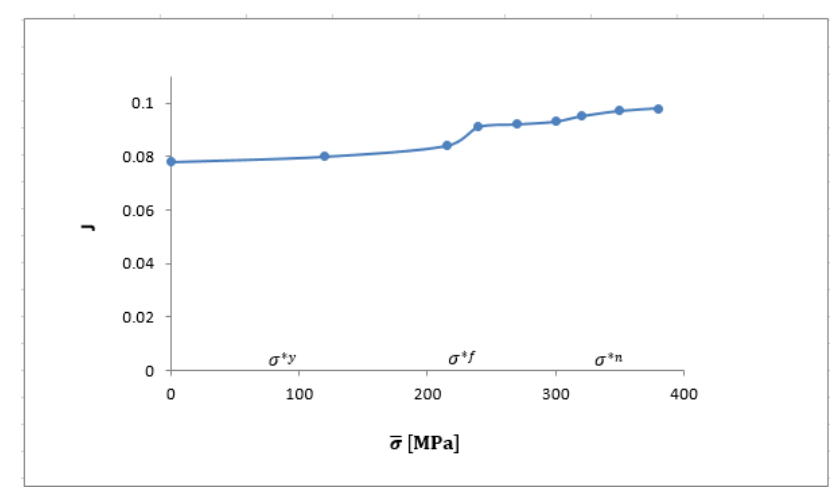

Figure 11. Stress-mode of presentation of J-parameter for corrosion-free specimen.

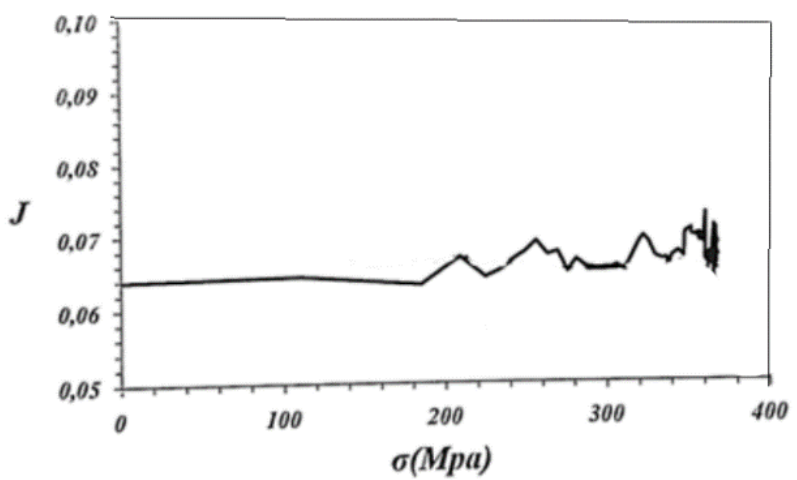

Figure 12. Stress-mode of presentation of J-parameter for 200 hours of corrosion.

However, as it can be seen in Figures (12-15) hydrogen accumulated by corrosion may lead to an appreciable change of the above described behavior of hydrogen-free J-parameter curve. One can so at first observe the "disappearance" of the sigmoidal transition behavior. This could happen because the hydrogen flowing into moving dislocation cores, by inducing stress-relief effects, may relax the magnetoelastic interaction and hence the pinning strength disfavoring so the transitional attachment of domain walls at moving dislocations systems. Further, it is observed that for corrosion times greater than $400 \mathrm{hrs}$ the earlier discussed quasiequilibrated behavior within the elastic stressing region is distorted by formation of a weak but distinctive minimalisation point within elastic stressing, far below the physical yielding $\sigma_{\mathrm{y}}{ }^{*}$. It is also observed in Figure (15) that for increased corrosion time of $1000 \mathrm{hrs}$, this maximalization is enhanced and the minimum point shifted toward higher elastic stresses. This behavior could be explained by the following combined influence approach: applied tensile stress enhances the preexisting, internal stress gradients by dynamic stress concentration (raiser) effects around grain boundaries, inclusiouns and other inhomogeneities. The enhanced stress gradient fields become now strong trapping sites for hydrogen atoms (ions) which by increasing their accumulation may form new intefaces in form of extensive atmospheres (clouds) or microvoids which now may act as high pinning energy barrier for moving domain walls. However, at the same time, the increased, opposite, hydrogen-induced stress- relief effect tend to relax these fields by lowering the energy barrier height. Thereafter, the macroscopical net result of the above combined applied stress and hydrogen influence on the micromagnetic activity is a balanced decrease and incrase in the J-signal by forming a minimum point. However, in this way, with increasing hydrogen accumulation the induced opposing void and stressrelief effects seems to equilibrate up to a certain level of increasing applied stress where again a minimum point is formed.

Furthermore, by this mode of stress presentation one can better observe and determine the onset and detailed occurrence of the earlier adopted Portervin - Le Chatelier - type micromagnetic process. For instance, one may observe more clearly that the 
ripple - type "modulation" arises close to physical yield point, at which, as earlier mentioned, grain - localized dislocation movement and multiplications sets on. The intensification of this process beyond the physical yield and close to cell flow stress point may be explained by the fact that around the latter point formation of massive dislocation configurations takes place at which more hydrogen may be attached and further transported. This seems to support the basic principle of the earlier adopted micromagnetic process by which moving dislocations may contribute to a correlated hydrogen - assisted domain wall transport.

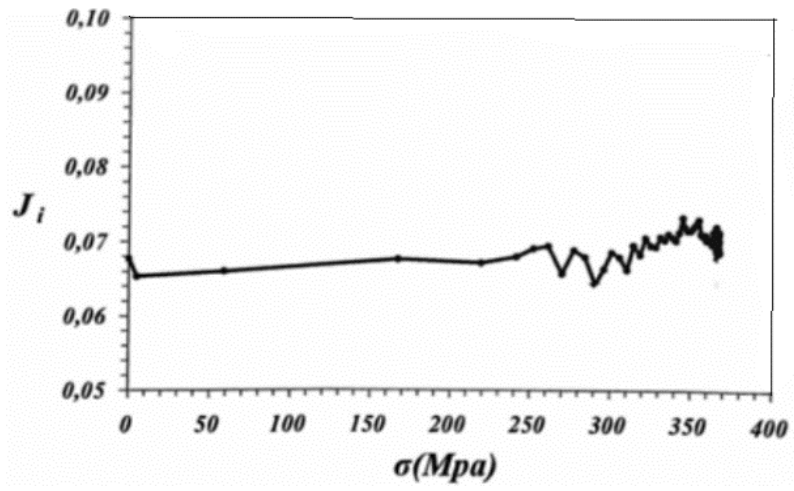

Figure 13. Stress-mode of presentation of J-parameter for 600 hours of corrosion.

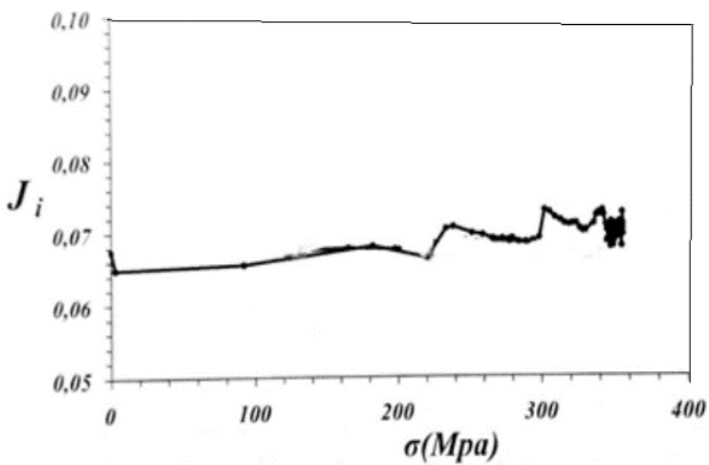

Figure 14. Stress-mode of presentation of J-parameter for 800 hours of corrosion.

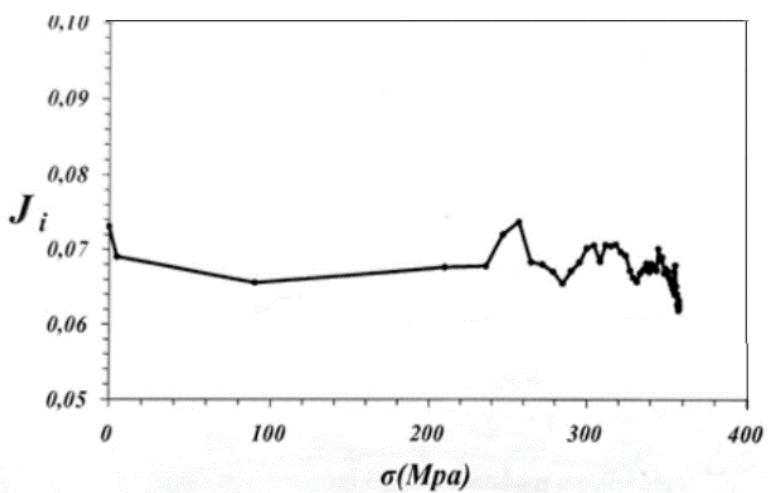

Figure 15. Stress-mode of presentation of J-parameter for 1000 hours of corrosion.

Moreover, by this mode of presentation a "hidden" fact may also be observed: for corrosion time 600 and 1000 hours, a relative change in the ripple "morphology" seem to occur determined by an associated decrease in the ripple number per unit stress or unit time as well as an increase in ripple amplitude. This may happen since above a "critical" corrosion time the cumulative hydrogen may promote the formation of respective large size void- structured Cottrell - Snoek type atmospheres (clouds) around moving dislocations [29]. This formation in turn, can be related with the initiation of range III of Figure2, and as such should exhibit an increased pinning strength for domain walls. This result in a longer immobilization time of domain walls at these atmospheres, allowing so an increased domain wall attachment on these atmospheres. Thus, by unpinning an increased number of domain walls are "set free" to move. In this way a comespouding increased change in the magnetic volume results, reflected by an increase in the ripple "amplitude" of Jparameter curve. At the same time, the increased immobilization time leads to a decrease in the related rate of pining/unpinning events, fact equivalent to a reduction in the number of ripples per unit stress or time and hence in the observed ripple "frequency" or "density".

\section{CONCLUSIONS}

Certain physical and mechanical microstructural properties of a low-carbon steel, determined by its mutual tensile elastoplastic and micromagnetic emission (ME) response, may considerable be influenced by cumulative atomic hydrogen produced under corrosive $\mathrm{NaCl}$-water solution environment. The influencing factors can better be revealed, described and analyzed by introducing a relevant specific ME- response parameter and employing certain intrinsic processes of ferromagnetic activity and mechanical behavior. In this manner one can show that an increase in the hydrogen accumulation with corrosion time leads to an associated increase of the embrittlement of steel, expressed by appreciable loss in its ductility as well as to a parallel increase in the magnetic hardening tendency related with a general reduced specific ME-response. The embrittlement and magnetic hardening are the net result of a highly localized effect of cumulative hydrogen consisting in two main mutual mechanisms: Void initiation - growth as well as stress-relief. As such, by adopting certain simple modelling approaches the dominating contribution to this effect of the first mechanism can reasonably be demonstrated. Under the influence of cumulative hydrogen the opposite and competitive action of applied stress and plastic strain-induced microstructural changes on the micromagnetic behavior can plausible be demonstrated. This is made by a combined approach of applied stress and plastic strain modes of presentation. As such, the delay effect of applied stress and the stimulating effect of plastic strain on the magnetic hardening tendency can be show. Certain subtle peculiarities of micromagnetic response behavior were able to be explained on the basis of a proposed more or less tentative Le Chatelier - Portervin-type micromagnetic process for which a relevant mechanism of moving dislocation-assisted correlated transport and redistribution of cumulative hydrogen and magnetic domain walls was assumed. Within the frame of the above findings the superior sensitivity of micromagnetic emission response, 
compared to mechanical one, in early detecting hydrogenassisted microstructural damages in steel under combined condition of applied stress and corrosive factors of $\mathrm{NaCl}$-water solution can be concluded.

\section{REFERENCES}

[1] H. Möller, E. T. Boshoff and H. Froneman; "The corrosion behavior of low carbon steel in natural and synthetic seawaters" The Journal of South African Institute of Mining and Metallurgy, Vol. 106, p. 585 - 592, 2006.

[2] A. W. Thomson and I. M. Bernstein, Advances in corrosion Science and Technology, vol. 17, R. W. staehle and M. G. Fontana, Eds. Plenum, New York, 1980, p. 53.

[3] Jingwei Zhao, Young Soo Chun and Cong Soo Lee; "Hydrogen embrittlement of low carbon steel during slow strain rate Test" Advanced Materials Research, Vols. 197 - 198, p. 642 - 645, 2011.

[4] A. K. Das, "Metallurgy of Failure Analysis", Mc Graw Hill, 1996.

[5] C. L. Briant, "Metallurgical Aspects of Environmental Failures", Materials Science Monographs, Vol 12, Elsevier, 1985.

[6] R. W. Hertzberg "Deformation and Fracture Mechanics of engineering materials" 3d Ed., Willey, 1989.

[7] D. O. Hagard and B. M. Trapnell, "Chemisorption", 2d Ed. Butterworth, Washington, DC, 1964.

[8] T. Alan Place, T. Srinivas Sudarhan, Cindy K. Waters and M. R. Louthan, Jr. "Fractographic Studies of the ductile - to - brittle Transition in Austenitic stainless steels", in Fractography of Modern engineering materials: Composites and Metals, Editors Masters, J and $\mathrm{Au}, \mathrm{J}$, ASTM 1987, p $350-365$.

[9] Yoneo Kikuta, Takao Araki and Toshio Kuroda, "Analysis of Fracture Morphology of Hydrogen assisted Cracking in steel and its Welds" in Fractography in Failure Analysis, ASTM STP 645, B. M. Strauss and W. H. Cullen, Jr. eds 1978, p $107-127$.

[10] R. P. Gangloff and R. P. Wei:" Fractographic analysis of gaseous hydrogen induced cracking in $18 \mathrm{~N}$ Maraging steel", in Fractography and Failure Analysis, Strauss/ Culler, Eds., ASTM-STP, 645, p 87-106,(1978).

[11] HG. R. Caskey, Jr.: "Hydrogen- induced brittle fracture of Type 304 L. Austentic stainless steel" in "Fractography and Materials Scince" Gilbertson/Zipp, Eds. ASTM-STP, 733, p 86-114,(1993)

[12] V. Provenzano, K. T'orr'ohen, D. Sturm and W. H. Culler, "Fractographic and Microstructural Analysis of StressCorrosion Cracking of ASTM, A-533 Grade B-class1 plate and ASTM, A-508 class 2 forging in pressurized Reactor-grade Water 93C", in Fractography and Materials Science, ASTM-STP-733, Gibbers ton /Zipp, Eds, 1981.
[13] Mein, D. A. and Bayles, R. A. " Fractographic Analysis of hydrogen-assisted cracking in Alpha - Beta Titanium alloys", in Fractography of Modern Engineering Materials, Composites and Metals ASTM-STP 948, P400, Masters/Au, Eds. (1987).

[14] West, A. J. and Hlbrook, J. H. in "Hydrogen effects in Metals", I. M. Bernstein and A. W. Thomson, Eds. American Jnst. of Mining s Metallurgical and Petroleum Engineering, p. 607, New York, 1981.

[15] Carber, R. and Bernstein, I. M. in "Environmental Degradation of Engineering Materials", M. R. Louthan, Jr. and R. P. Mc Nitt, Eds., Virginia Polytechnic Institute, Blacksburg, VA, 1977, p. 463.

[16] May L. Martin, Mohsen Dadfarnia, Akihide Nago, Shuai Wanag and Petros Sofronis "Enumeration of hydrogen on hanced loclized plasticitg mechanisms for hydrogen embritzlement in structural steels" Acta Materialia, Vol. 156, February 2019, pp. 734-750.

[17] Mohamad Blaow, Jahn Terence and Brian A. Shaw, "The effect of microstructure and applied stress on magnetic Barkhausen emission in induction hardened steel, J. Mat. Sci. Val 42, p. 4364-4371, (2007).

[18] C. E. Stefanita, L. Clapham and D. L. Atherton, "Subtle changes in magnetic Barkhausen noise before the macroscopic elastic limit” J. Mat. sc. 35, p 2675-(2000).

[19] P. Polukhin, S. Gorelic and V. Vorontsov, "Physical Principles of Plastic Deformation", (in English), Mir Publishers, Moscow. 1983.

[20] Thomas W. Krause, L. CLAPHAM, Andreas Pttantyus and Davied L. Atherton," Investigation on the stressdependent magnetic easy axis in steel using magnetic Barkhausen noise”. J. Appl. phys. Vol. 79 (8), 15 April 1996, p 4242-4252.

[21] H. Träuble in "Moderne Probleme der Metallphysik", A Seeger Ed. Springer Verlag 1966.

[22] S. Chikazumi, Physics of Ferromagnetis, Oxford, Science Publications Reprint 2005.

[23] A. Hubert and R. Schäfer: "Magnetic Domains: The Analysis of Magnetic Microstructure", Springer, Corrected Printing 2000.

[24] D. C. Jilles and D. L. Atherton, "Theory of the magnetization process in ferromagnets and its application to the magnetomechanical effect" J. Phys. D., 17 (1984), p. 1265 - 1281.

[25] V. N. Kytopoulos, A. Altzoumailis, Chr. Panagopoulos, Chr. Riga. "Hydrogen influence on certain mechanical and magnetic properties of a stressed Low-Carbon Steel after Corrosion in NaCl-water solution", Procedia Structural Integrity 26 (2020), p. p. 113-119. www.sciencedirect.com

[26] D Gwang and $\mathrm{H} \mathrm{C} \mathrm{Kim} \mathrm{the} \mathrm{influence} \mathrm{of} \mathrm{plastc}$ deformations on Barkhausen effects and magnetic properties in mild steel Journal of Phys D applied physics, 198821 p $1807-1813$. 
[27] Tadao Nozawa, Masato Mizogami, Hisai Mogi and Yukio Matsuo, 'Domain structures and magnetic properties of advanced grain- oriented silicon steel" J. Magn. Mater. 133, (1994) p. 115-122.

[28] E. K. Ioakeimidis, V. N. Kytopoulos, E. Hristoforou "Investigation of magnetic, mechanical and micro failure behavior of ARMCO - type low carbon steel corroded in $3.5 \% \mathrm{NaCl}$-aqueous solution"

Materials Science and Engineering: A, Volume 583, 2013, p 254-260.

[29] J. Degauque, B. Astie and L. P. Kubin,'Evidence for
Interaction between Magnetic domain walls and Dislocations in high-purity Iron from Magnetomechanical damping measurements." Phys. state so. (a), 45, p 493-501 (1978).

[30] Martha Pardavi-Horvath, "Magnetic Noise Barkhausen Effect "in Wiley Encyclopedia of Electrical and Electronic Engineering, Val. 12, p. 52-64, y. E. Webster Ed., 1999.

\section{Creative Commons Attribution License 4.0} (Attribution 4.0 International, CC BY 4.0)

This article is published under the terms of the Creative Commons Attribution License 4.0

https://creativecommons.org/licenses/by/4.0/deed.en_US 